\title{
Basic and Translational Science
}

\section{Improved Penile Histology}

by Phalloidin Stain: Circular and

Longitudinal Cavernous Smooth

Muscles, Dual-endothelium Arteries, and
Erectile Dysfunction-associated Changes

\author{
Guiting Lin, Xuefeng Qiu, Thomas M. Fandel, Maarten Albersen, Zhong Wang, \\ Tom F. Lue, and Ching-Shwun Lin
}

OBJECTIVE

To investigate whether fluorochrome-conjugated phalloidin can delineate cavernous smooth muscle (CSM) cells and whether it can be combined with immunofluorescence (IF) staining to quantify erectile dysfunction (ED)-associated changes.

METHODS

ED was induced by cavernous nerve crush in rats. Penile tissues of control and ED rats were stained with Alexa-488-conjugated phalloidin and/or with antibodies against rat endothelial cell antigen (RECA), CD31, neuronal nitric oxide synthase (nNOS), and collagen-IV (Col-IV).

RESULTS Phalloidin was able to delineate CSM as composed of a circular and a longitudinal compartment. When combined with IF stain for CD31 or RECA, it helped the identification of the helicine arteries as covered by endothelial cells on both sides of the smooth muscle layer. When combined with IF stain for nNOS, it helped the identification that nNOS-positive nerves were primarily localized within the dorsal nerves and in the adventitia of dorsal arteries. When combined with IF stain for Col-IV, it helped identify that Col-IV was localized around smooth muscles and beneath the endothelium. Phalloidin also facilitated the quantitative analysis of ED-related changes in the penis. In rats with cavernous nerve injury, RECA or Col-IV expression did not change significantly, but CSM and nNOS nerve contents decreased significantly.

CONCLUSION Phalloidin stain improved penile histology, enabling the visualization of the circular and longitudinal compartments in the CSM. It also worked synergistically with IF stain, permitting the visualization of the dual endothelial covering in helicine arteries, and facilitating the quantification of ED-related histologic changes. UROLOGY 78: 970.e1-970.e8, 2011. (c) 2011 Elsevier Inc.

$\mathrm{V}$ isualization of smooth muscle in the penis is usually performed by immunohistochemistry (IHC) or immunofluorescence (IF) with an anti-smooth muscle actin (anti-SMA) antibody. For example, Ferrini et $\mathrm{al}^{1}$ and Yang et $\mathrm{al}^{2}$ have used IHC and IF, respectively, with anti-SMA antibody to visualize the smooth muscle structure

Funding Support: This work was supported by grants from the Arthur Rock Foundation and the National Institutes of Health (DK045370).

Maarten Albersen is a fellow of the Research Foundation-Flanders (FWO).

From the Knuppe Molecular Urology Laboratory, Department of Urology, University of California, San Francisco, CA; Department of Urology, Affiliated Drum Tower Hospital, Medical School of Nanjing University, Nanjing, China; Department of Urology, University Hospitals, Leuven, Belgium; and Department of Urology, Ninth People's Hospital, Affiliated to Medical College of Shanghai Jiao-Tong University, Shanghai, China

Reprint requests: Dr. Ching-Shwun Lin, Department of Urology, University of California, 533 Parnassus Ave, Box 0738, San Francisco, CA 94143-0738. E-mail: clin@urology.ucsf.edu

Submitted: January 14, 2011, accepted (with revisions): June 6, 2011 in rat penile tissues. Another commonly used method, the trichrome stain, has the additional benefit of being able to discriminate between smooth muscle and collagen, and this was previously used by Ferrini et $\mathrm{al}^{1}$ to quantify corporal muscle and collagen contents.

Phalloidin is a toxin from the toadstool "Death Cap" (Amanita phalloides), and its potentially lethal effect was caused by its ability to bind actin and prevent actin depolymerization. ${ }^{3,4}$ Because of its small size, phalloidin can easily penetrate into the densely packed actin network, and its stabilizing action on actin filaments further makes it an ideal probe for the detection of actins. ${ }^{5}$ Thus, several fluorochrome-conjugated derivatives of phalloidin have been used for the visualization of cellular actin. ${ }^{6,7}$ In particular, the Alexa-conjugated phalloidin derivatives have been shown to yield brightness and photostability that are superior to all other spectrally similar conjugates. ${ }^{8}$ For example, Alexa-488-conjugated 
phalloidin has been used to generate sharp images of aortic smooth muscle cells (SMCs). ${ }^{9}$

In the present study we used Alexa-488-conjugated phalloidin to stain the penises of rats. The technique allowed us to clearly visualize not only the individual SMC but also their relationship with the endothelium, nerves, and extracellular components when combined with IF for these latter structures. As a consequence, we recognized that the cavernous smooth muscles (CSM) is composed of 2 layers - circular and longitudinal. We also discovered that small blood vessels (helicine arteries) within the cavernous tissue are lined with 2 layers of endothelial cells_-one on the luminal side and another on the abluminal side of the smooth muscle layer. Finally, we found that the combined phalloidin/IF stain could be used to obtain valuable information on the histologic changes associated with cavernous nerve $(\mathrm{CN})$ injury.

\section{MATERIAL AND METHODS}

\section{Animals}

All experimental protocols were approved by the Institutional Animal Care and Use Committee at University of California, San Francisco. Sixteen 3-month-old male Sprague-Dawley rats obtained from Charles River Laboratories (Wilmington, MA) were randomized into 2 equal groups and treated as follows. Briefly, under $2 \%$ isoflurane anesthesia, a lower abdomen midline incision was made and the prostate gland exposed. The $\mathrm{CNs}$ and major pelvic ganglia were then identified posterolaterally on both sides of the prostate. In the control (C) group, no further manipulation was performed except for closing the wound. In the nerve crush (NC) group the CNs were isolated and crushed for 2 minutes per side, using a dedicated needle holder. ${ }^{10}$ The abdomen was then closed in 2 layers. Four months later, erectile function was determined by measurement of intracavernous pressure (ICP) during electrostimulation of CNs. All C rats had normal ICP, whereas all $\mathrm{NC}$ rats had reduced ICP. ${ }^{10}$

\section{Phalloidin and Immunofluorescence Stain}

Rat penises were harvested and fixed in cold $2 \%$ formaldehyde and $0.002 \%$ saturated picric acid in $0.1-\mathrm{M}$ phosphate buffer, $\mathrm{pH}$ 8.0, for 4 hours followed by overnight immersion in buffer containing $30 \%$ sucrose. The specimens were then embedded in optimum cutting temperature compound (Sakura Finetek, United States, Torrance, CA) and stored at $-70^{\circ} \mathrm{C}$ until use. Fixed frozen tissue specimens were cut at $5 \mu \mathrm{m}$, mounted onto SuperFrost-plus charged slides (Fisher Scientific, Pittsburgh, $\mathrm{PA}$ ), and air dried for 5 minutes. To stain F-actin, tissue sections were incubated with Alexa-488-conjugated phalloidin (1:100 in $1 \%$ bovine serum albumin, Invitrogen, Carlsbad, CA) for 20 minutes at room temperature, followed by incubation with 4',6-diamidino-2-phenylindole (DAPI, for nuclear staining, $1 \mu \mathrm{g} / \mathrm{mL}$, Sigma-Aldrich, St. Louis, MO). For IF stain, tissue sections were placed in $4 \%$ paraformaldehyde for 10 minutes, washed twice in phosphate-buffered saline (PBS) for 5 minutes, and incubated with $3 \%$ horse serum in PBS/0.3\% Triton X-100 for 30 minutes at room temperature. After draining this solution from the tissue section, the slides were incubated at $4^{\circ} \mathrm{C}$ with mouse anti-rat endothelial cell antigen (RECA; AbD Serotec, Raleigh, NC), mouse anti-CD31 (Millipore, Billerica, MA), rabbit anti-Collagen IV (Abcam, Inc.,
Cambridge, MA), or rabbit anti-neuronal nitric oxide synthase (nNOS) (Santa Cruz Biotechnology, Santa Cruz, CA). Control tissue sections were similarly prepared, except no primary antibody was added. After rinsing the sections in PBS, they were incubated with Alexa-488 or Alexa-594-conjugated goat antirabbit or goat anti-mouse secondary antibodies (Invitrogen, Carlsbad, CA). After rinsing in PBS, the slides were further stationed with DAPI. When indicated, the immunostained tissues were also stained with Alexa-488-conjugated phalloidin as described previously before the DAPI stain.

\section{Image Analysis and Quantification}

The stained tissues were examined with a Nikon Eclipse E600 fluorescence microscope and photographed with Retiga 1300 Qimaging camera using the ACT-1 software (Nikon Instruments, Inc., Melville, NY). Individual images generated from the green, red, and blue channels were superimposed to generate the composite figures. Computerized histomorphometric analysis was performed using Image-plus 5.1 software (Media Cybernetics, Bethesda, MD). To analyze CSM content, the corpus cavernosum was photographed at $20 \mathrm{x}$ magnification, and the ratio between the phalloidin-stained area (pixel number of green stain) and the entire corpus cavernosum (pixel number of areas enclosed within the tunica albuginea) was calculated. To analyze nNOS content, the number of positively stained dots within dorsal nerves was counted at 200x magnification. To analyze endothelial or collagen-IV (Col-IV) content, the integrated optical density of positively stained area was obtained at 100x magnification.

\section{Statistical analysis}

Data were analyzed with Prism 4 (GraphPad Software, San Diego, $\mathrm{CA})$. Analysis of variance was used to determine the difference between the means of different treatment groups, followed by the paired $t$-test. Difference was considered significant when $P<.05$. All data are shown as mean \pm standard deviation.

\section{RESULTS}

\section{Circular and Longitudinal CSMs}

Alexa-488-conjugated phalloidin intensely stained smooth muscles in the rat penis, including the 2 dorsal arteries, the dorsal vein, and the CSM (Fig. 1A). At higher magnifications, it became clear that the CSM is divided into a circular and a longitudinal compartment (Fig. 1B, 1C). The circular CSM, which varies in thickness from 1-6 layers (mostly $2-3$ ), is located closer to the connective tissue and distributed along the walls of the cavernous sinusoids. The longitudinal CSM, which appears as clusters of SMC in crosssectional views, runs parallel to the length of the penis. In contrast to its circular counterpart, the number of cells in the longitudinal CSM clusters varies greatly from a few cells to several dozen. To illustrate the improved histology with phalloidin stain, a side-by-side comparison of phalloidin and actin shows that CSM fibers appeared coarse and granular when stained with anti-SMA antibody (Fig. 1D, 1E).

\section{The Endothelium}

The delineation of smooth muscle by phalloidin stain permitted a clear visualization of the relationship between smooth muscle and the endothelium-the latter 

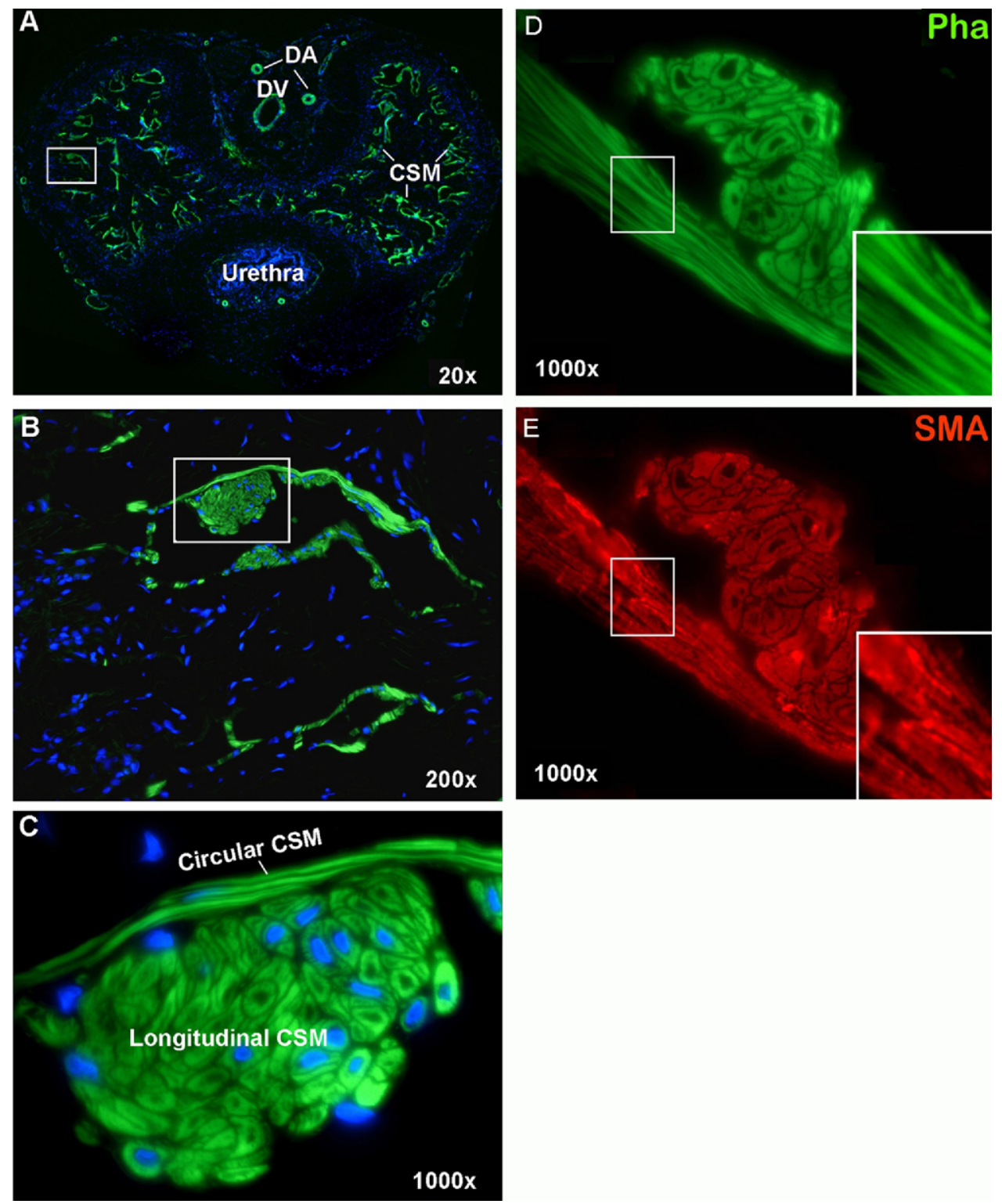

Figure 1. Smooth muscles in rat penis. Rat penis was stained with Alexa-488-conjugated phalloidin, which specifically detected smooth muscles (green stains) in dorsal arteries (DA), dorsal vein (DV), and cavernous tissue (CSM). Boxed areas in $(\mathbf{A})$ and $(\mathbf{B})$ are sequentially enlarged in $(\mathbf{B})$ and $(\mathbf{C})$, respectively. These tissue sections were costained with DAPI for the visualization of cell nuclei (blue). The seemingly "missing nuclei" (phalloidin-stained cells without DAPI) is a result of tissue sectioning at an angle or plane that missed the nuclei. Panels (D) and (E) are consecutive sections stained with phalloidin and anti-SMA, respectively. Inserts are images enlarged digitally from the boxed areas.

defined by IF staining with anti-RECA and anti-CD31 antibodies (Fig. 2). Throughout the cavernous sinusoids, all musculatures are covered with a layer of endothelial cells on their sinusoidal side. In addition, a few blood vessel-like structures (helicine arteries) can be seen in the vicinity of the longitudinal CSM (Fig. 2A, 2C, 2D). Surprisingly, these vessel-like structures are covered by 2 layers of endothelial cells-one on the luminal and another on the abluminal side of the smooth muscle. It is unlikely that such a dualendothelium structure is a staining artifact because dorsal arteries and dorsal vein in the same tissue sec- tion as the one in Fig. 2C were stained positive for RECA only on the luminal side (Fig. 2E).

\section{Nerves}

The delineation of smooth muscle by phalloidin stain also permitted the clear visualization of the relationship between smooth muscle and nNOS-positive nerves (Fig. 3). First, nNOS protein was primarily localized within the dorsal nerves, which were devoid of phalloidin stain (Fig. 3A). Second, nNOS protein was also localized in the adventitia of dorsal arteries, whose smooth muscle was positively stained by phal- 

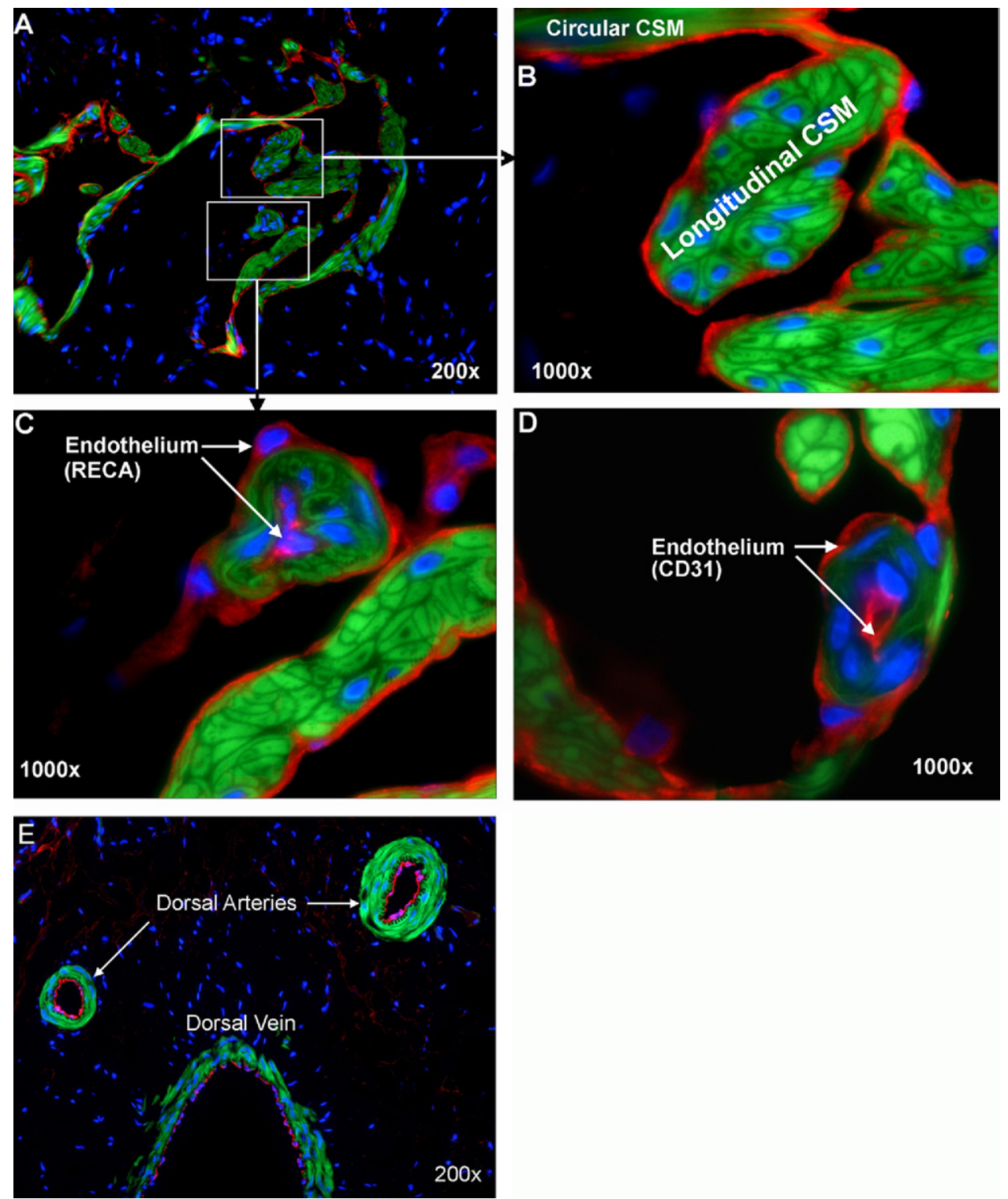

Figure 2. Endothelium in rat penis. Rat penis was stained with Alexa-488-conjugated phalloidin and with Alexa-594conjugated anti-RECA $(\mathbf{A}-\mathbf{C})$ or anti-CD31 antibody $(\mathbf{C})$. These stains provide visualization of smooth muscle in green and the endothelium in red. Boxed areas in $(\mathbf{A})$ are enlarged in $(\mathbf{B})$ and $(\mathbf{C})$, respectively. Arrows in $(\mathbf{C})$ and $(\mathbf{D})$ point to the internal and external endothelia (red) of helicine arteries. (E) Dorsal arteries and vein that were stained positive for RECA (red) in the luminal side but not the abluminal side of smooth muscle (green). All tissue sections were costained with DAPI for the visualization of cell nuclei (b/ue).

loidin (Fig. 3B). Finally, nNOS protein was also localized at a relatively low level to the periphery of CSM bundles (Fig. 3C).

\section{Extracellular Matrix}

The cavernous extracellular matrix (ECM) is composed mainly of collagen types I, III, and IV. ${ }^{11}$ Because of Col-IV's close association with SMCs, ${ }^{12}$ it was examined in the present study by IF staining in conjunction with the phalloidin stain for CSM. Col-IV was seen surrounding each longitudinal SMC and sandwiched between circular CSM fibers (Fig. 4A, 4B). It was also localized on the sinusoidal side of muscle bundles, seemingly forming the endothelial basement membrane (Fig. 4A, 4B). The relationship between this basement membrane-like structure and the endothelium was further examined by double staining with anti-RECA antibody (Fig. 4C-F). To ensure specificity, the endothelium and Col-IV were alternately stained with green and red fluorochromes. The results show that, in regard to the longitudinal CSM bundles, Col-IV was seen more internally (surrounding muscle cells), whereas the endothelium was visualized externally (covering the smooth muscle bundle) (Fig. 4C, 4D). In regard to the vessel-like structures (helicine arteries), Col-IV is located between the endothelium and the smooth muscle (Fig. 4E, 4F). 

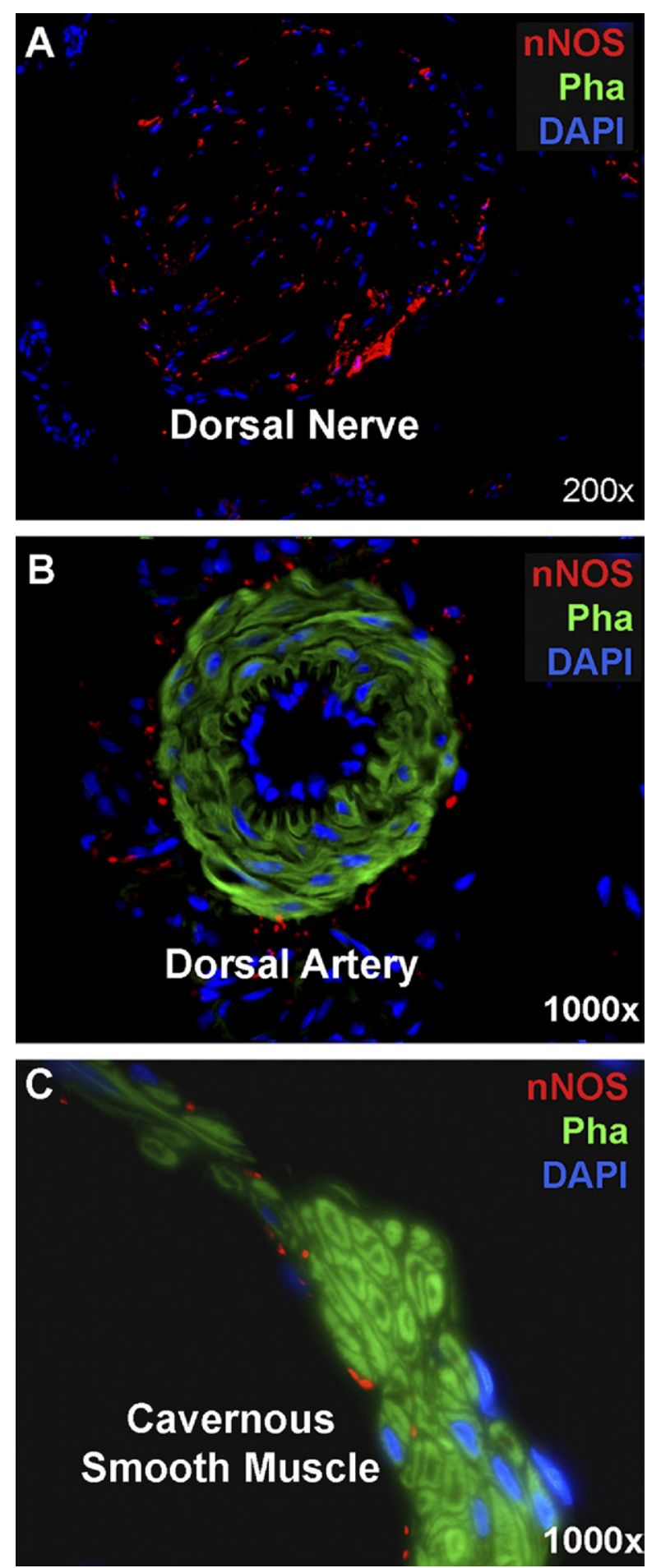

Figure 3. nNOS-positive nerves in rat penis. Rat penis was stained with Alexa-488-conjugated phalloidin and with Alexa-594-conjugated anti-nNOS. These stains provide visualization of smooth muscles in green and nNOS-positive nerves in red. (A) A representative dorsal nerve stained positive for nNOS but negative for smooth muscle. Blue stains are Schwann cell nuclei. (B) A representative dorsal artery with its smooth muscle stained positive by phalloidin. Red stains surrounding the smooth muscle are nNOS-positive nerves. Blue stains encircling the lumen are endothelial cell nuclei. (C) A representative CSM bundle with a few nNOS-positive nerves innervating at its periphery.

\section{Quantification of Erectile Dysfunction-Related Changes}

To demonstrate the utility of the improved penile histology afforded by phalloidin stain, we quantified changes in the penis of a $\mathrm{CN}$ injury erectile dysfunction (ED) model. The results show that the CSM content (\% of total area) was significantly reduced in ED compared with normal rats $(4.51 \pm 0.57$ vs $9.47 \pm 0.96, P<.01)$. Likewise, the number of nNOS-positive nerves within dorsal nerves was also significantly reduced in ED when compared with normal rats $(213.8 \pm 38.6$ vs $604.3 \pm 68.7$, $P<.01$ ). Significant reduction in Col-IV content (measured as integrated optical density) was also found in ED rats $(100443.9 \pm 21023.7$ vs $140322.8 \pm 19012.9$, $P<.05)$. In regard to the cavernous endothelial content (measured as integrated optical density), no statistical difference between ED and normal rats was found $(63078.5 \pm 4948.5$ vs $70451.2 \pm 6742.6, P>.05)$ at 4 months after $\mathrm{CN}$ injury.

\section{COMMENT}

Phalloidin is a small molecule that can penetrate rapidly into the tightly packed cytoskeleton. This property and its highly specific affinity for actin make it an ideal probe for actin, and to this end, several fluorochrome-conjugated phalloidin derivatives have been used to stain smooth muscle in various organs and tissues. ${ }^{9,13}$ The staining procedure is applicable to both frozen and paraffin-embedded tissues ${ }^{14}$ and can be completed in 30 minutes, in contrast to IF, which may require as long as 24 hours. As shown in the present study, phalloidin stain works synergistically with IF for various antigens, including CD31, nNOS, and Col-IV. In contrast, double-IF staining requires that 2 primary antibodies be generated from 2 different host species to incubate the antibodies together. ${ }^{15,16}$ We have observed that double-IF staining occasionally produced low-quality histology because of interference between the primary antibodies and/or between the secondary antibodies.

Phalloidin has been used for assessing smooth muscle content in a $\mathrm{CN}$ ablation rat model. ${ }^{17}$ However, histologic images in this study are not as sharp as what is shown in the present study. One possible explanation is that the earlier study used tetramethyl-rhodamine isothiocyanate-conjugated phalloidin, which has been shown to produce lesser quality images when compared with Alexa-conjugated phalloidin. ${ }^{8}$ The exceptionally high optical resolution afforded by Alexa-conjugated phalloidin enabled us to recognize that the CSM was divided into a circular and a longitudinal compartmentcharacteristic structures of tubular organs, such as intestines, urinary bladder, and urethra. Thus, the penile corpus cavernosum can possibly be considered as a multiunit tubular organ, with each unit (sinusoid) consisting of a circular and a longitudinal smooth muscle. The circular CSM regulates the penile girth and the longitudinal CSM regulates the length during tumescence and detumescence. 

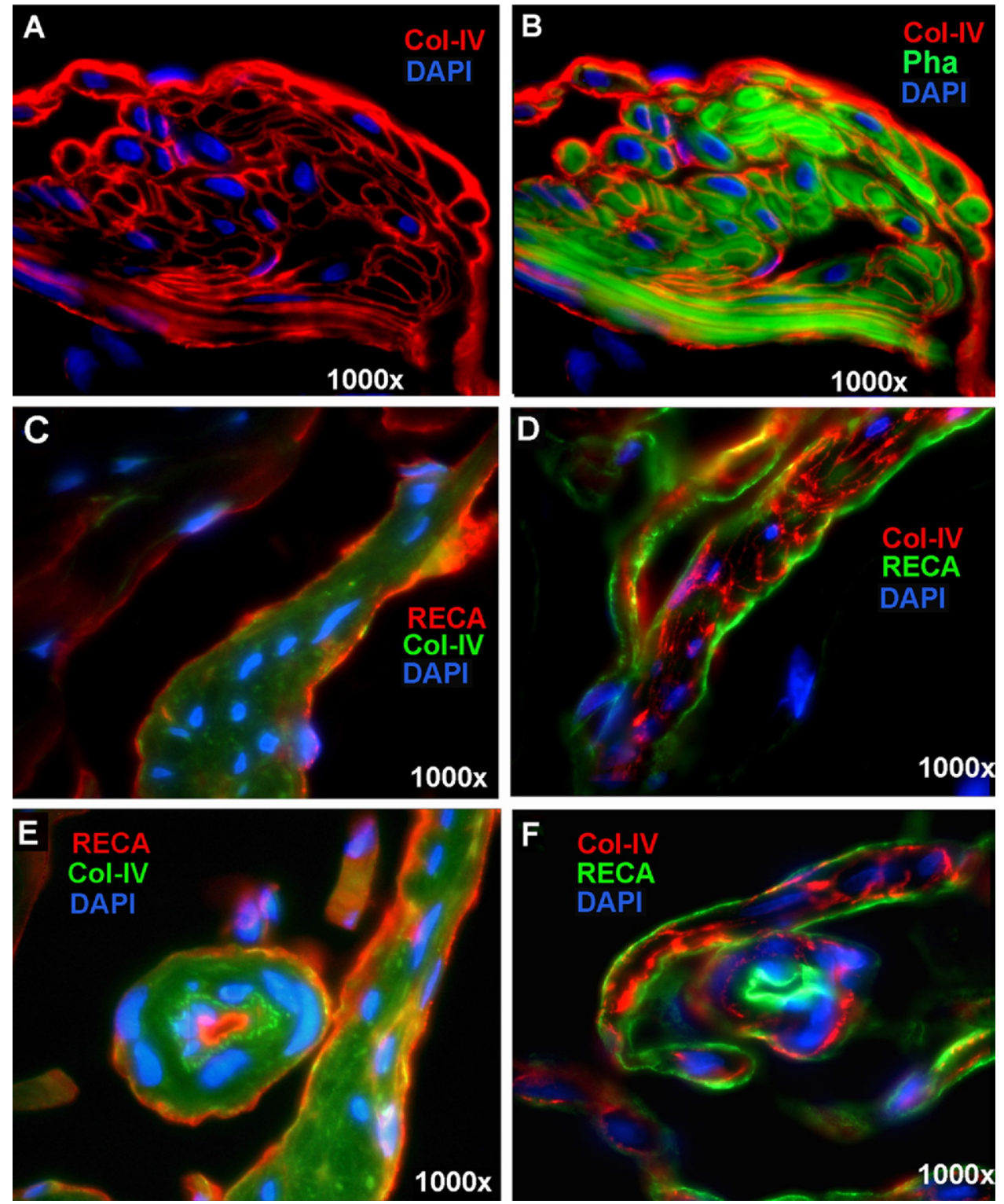

Figure 4. Col-IV in rat penis. (A, B) Rat penis stained with Alexa-488-conjugated phalloidin and with Alexa-594-conjugated anti-Col-IV antibody. For enhanced clarity, Col-IV expression was shown in the absence of phalloidin stain in (A). For visualizing the relationship between Col-IV and SMC, phalloidin-stained image was added in (B). (C, E) Rat penis stained with Alexa-488-conjugated Col-IV (green) and with Alexa-594-conjugated anti-RECA (red). (D, F) Rat penis stained with Alexa-488-conjugated anti-RECA (green) and with Alexa-594-conjugated anti-Col-IV (red). Note that (E) and (F) show a helicine artery in the center. All tissue sections were costained with DAPI for the visualization of cell nuclei (blue).

It is well known that each penile cavernous sinusoid is an equivalent of a blood vessel's lumen with both having a single layer of endothelial cells covering the luminal side of the smooth muscle layer. The endothelial cells can be easily identified by IF using either anti-RECA (highly specific for rat endothelial cells) or anti-CD31, as demonstrated in the present study and in many previously published studies. ${ }^{18,19}$ However, in the present study we saw that certain smooth muscle bundles that otherwise resembled the longitudinal CSM contained centrally located RECA or CD31-positive cells. On the basis of this observation, we propose that these are helicine arteries entering the cavernous sinusoids; while retaining the arterial endothelium on the luminal side, they also become covered by the cavernous endothelium on the abluminal side. Thus, it appears that the vascular and cavernous smooth muscle cells are "protected" from direct contact with the blood by the endothelium. This hypothesis is based on experiments in which de-endothelialization leads to platelet activation and aggregation ${ }^{20}$ as well as SMC proliferation. ${ }^{21}$

Burnett et $\mathrm{al}^{22}$ were the first to demonstrate the localization of nNOS-positive nerves in the penis. They used immunohistochemical (IHC) stain to show that these nerves were mainly localized in the dorsal nerves and were also visible near the dorsal arteries and in the cavernous tissue. These findings are confirmed in the present study; in addition, our phalloidin stain enabled 
the visualization of the relationship between nNOSpositive fibers and smooth muscles in the dorsal arteries and the cavernous tissue. Specifically, these nerves were located outside of the arterial smooth muscle and therefore in the adventitia of the dorsal artery. Furthermore, their dotted appearance in cross-sectional view suggests an orientation parallel to the muscle fibers in the artery as well as in the cavernous tissue.

The cavernous ECM is composed mainly of collagen types I, III, and IV, with types I and IV being predominant. ${ }^{11}$ Although many studies have examined collagen expression in the penis, few have looked into the specific localization of each collagen type. Five of these reports, all from the same group of researchers, used IHC to examine differences in Col-III expression between hypertensive and control animals. ${ }^{23,24}$ To our knowledge, there has been no report that examined the localization of Col-IV in the penis. In blood vessels, Col-IV is a major component of the endothelial basement membrane and of the basal lamina surrounding SMCs; it also plays important roles in maintaining the contractile phenotype of SMC. ${ }^{12}$ Thus, because the present study's main objective is to use the phalloidin stain to visualize the penile SMC and its surroundings, Col-IV was chosen to represent the cavernous ECM. Indeed, the results show that Col-IV was localized to the subendothelial area as well as in the surroundings of individual SMCs. Very little Col-IV expression was found in the rest of the cavernous tissue, which is otherwise the main locale of collagen expression. That is, Col-IV is primarily associated with the endothelium and CSM and Col-I and Col-III are associated with the remaining areas of the cavernous tissue.

We have previously published a rat ED model that simulates postprostatectomy nerve injury. ${ }^{10}$ The penile tissue samples of this study were used in the present study for the purpose of demonstrating the use of the phalloidin stain. The results show that the CSM and nNOS nerves were significantly reduced in rats with $\mathrm{CN}$ injury, whereas no statistical difference was found for the endothelium at 4 months after injury. In regard to Col-IV, although the absolute amount of Col-IV was lower, the relative amount to CSM was actually increased.

In addition to nerve injury, we have found that the phalloidin stain to be useful for the evaluation of other types of $\mathrm{ED}$, including diabetes-associated $\mathrm{ED}^{25}$ and hyperlipidemia-associated ED. ${ }^{26}$

\section{CONCLUSIONS}

Phalloidin stain improves penile histology, enabling the visualization of the circular and longitudinal arrangements of CSM. It also provides synergism with IF stain, enabling the identification of the helicine artery's twolayer endothelium and the quantification of ED-related histologic changes in the penis.

\section{References}

1. Ferrini MG, Kovanecz I, Sanchez S, et al. Fibrosis and loss of smooth muscle in the corpora cavernosa precede corporal venoocclusive dysfunction (CVOD) induced by experimental cavernosal nerve damage in the rat. J Sex Med. 2009;6:415-428.

2. Yang R, Huang YC, Lin G, et al. Lack of direct androgen regulation of PDE5 expression. Biochem Biophys Res Commun. 2009;380:758762.

3. Löw I, Wieland T. The interaction of phalloidin. Some of its derivatives, and of other cyclic peptides with muscle actin as studied by viscosimetry. FEBS Lett. 1974;44:340-343.

4. Wehland J, Osborn M, Weber K. Phalloidin-induced actin polymerization in the cytoplasm of cultured cells interferes with cell locomotion and growth. Proc Natl Acad Sci U S A. 1977;74:5613. 5617.

5. Small JV, Zobeley S, Rinnerthaler G, et al. Coumarin-phalloidin: a new actin probe permitting triple immunofluorescence microscopy of the cytoskeleton. J Cell Sci. 1988;89(1):21-24.

6. Wulf E, Deboben A, Bautz FA, et al. Fluorescent phallotoxin, a tool for the visualization of cellular actin. Proc Natl Acad Sci U S A. 1979;76:4498-4502.

7. Faulstich H, Zobeley S, Rinnerthaler G, et al. Fluorescent phallotoxins as probes for filamentous actin. J Muscle Res Cell Motil. 1988;9:370-383.

8. Panchuk-Voloshina N, Haugland RP, Bishop-Stewart J, et al. Alexa dyes, a series of new fluorescent dyes that yield exceptionally bright, photostable conjugates. J Histochem Cytochem. 1999;47: 1179-1188.

9. Black J, Dykes A, Thatcher S, et al. FRET analysis of actin-myosin interaction in contracting rat aortic smooth muscle. Can J Physiol Pharmacol. 2009;87:327-336.

10. Fandel TM, Bella AJ, Lin G, et al. Intracavernous growth differentiation factor- 5 therapy enhances the recovery of erectile function in a rat model of cavernous nerve injury. J Sex Med. 2008;5: 1866-1875.

11. Luangkhot R, Rutchik S, Agarwal V, et al. Collagen alterations in the corpus cavernosum of men with sexual dysfunction. J Urol. 1992;148:467-471.

12. Barnes MJ, Farndale RW. Collagens and atherosclerosis. Exp Gerontol. 1999;34:513-525.

13. Kwon O, Phillips CL, Molitoris BA. Ischemia induces alterations in actin filaments in renal vascular smooth muscle cells. Am J Physiol Ren Physiol. 2002;282:F1012-F1019.

14. Swamynathan SK, Crawford MA, Robison WG Jr., et al. Adaptive differences in the structure and macromolecular compositions of the air and water corneas of the "four-eyed" fish (Anableps Anableps). FASEB J. 2003;17:1996-2005.

15. Mason DY, Micklem K, Jones M. Double immunofluorescence labelling of routinely processed paraffin sections. J Pathol. 2000;191: 452-461.

16. Bzorek M, Stamp IM, Petersen BL, et al. Use of commercially available rabbit monoclonal antibodies for immunofluorescence double staining. Appl Immunohistochem Mol Morphol. 2008;16:387392.

17. Hu WL, Hu LQ, Song J, et al. Fibrosis of corpus cavernosum in animals following cavernous nerve ablation. Asian J Androl. 2004; 6:111-116.

18. Song YS, Lee HJ, Park IH, et al. Human neural crest stem cells transplanted in rat penile corpus cavernosum to repair erectile dysfunction. BJU Int. 2008;102:220-224; [Discussion:224].

19. Ning H, Liu G, Lin G, et al. Fibroblast growth factor 2 promotes endothelial differentiation of adipose tissue-derived stem cells. J Sex Med. 2009;6:967-979.

20. Stemerman MB, Ross R. Experimental arteriosclerosis. I. Fibrous plaque formation in primates, an electron microscope study. J Exp Med. 1972;136:769-789. 
21. Jacot JG, Wong JY. Endothelial injury induces vascular smooth muscle cell proliferation in highly localized regions of a direct contact co-culture system. Cell Biochem Biophys. 2008;52:37-46.

22. Burnett AL, Lowenstein CJ, Bredt DS, et al. Nitric oxide: a physiologic mediator of penile erection. Science. 1992;257:401403.

23. Mazza ON, Angerosa M, Becher E, et al. Differences between candesartan and hydralazine in the protection of penile structures in spontaneously hypertensive rats. J Sex Med. 2006;3:604611.
24. Toblli JE, Cao G, Casas G, et al. In vivo and in vitro effects of nebivolol on penile structures in hypertensive rats. Am J Hypertens. 2006;19:1226-1232.

25. Albersen M, Lin G, Fandel TM, et al. Functional, metabolic and morphological characteristics of a novel rat model of type 2 diabetes-associated erectile dysfunction. Urology. 2011 [epub ahead of print].

26. Qiu X, Fandel TM, Lin G, et al. Cavernous smooth muscle hyperplasia in a rat model of hyperlipidemia-associated erectile dysfunction. BJU Int. 2011; In press. 